\title{
Role of collateral embolization in addition to uterine artery embolization followed by hysteroscopic curettage for the management of cesarean scar pregnancy
}

Guodong Zhang, Jijun Li, Jun Tang, Lei Zhang, Dechao Wang and Zengtao Sun *id

\begin{abstract}
Background: The aim of this study was to assess the feasibility, safety and outcome of the embolization of nongonadal collateral supplying gestational sac (GS) in addition to uterine artery embolization (UAE), followed by hysteroscopic curettage for the management of cesarean scar pregnancy (CSP).

Methods: A retrospective study was undertaken from January 2012 to September 2018 in 24 CSP patients in whom non-gonadal collaterals supplying GS were identified by arterial angiography performed immediately after UAE. These patients underwent attempt collateral embolization in addition to UAE, followed by hysteroscopic curettage for the management of CSP. The 24 patients were divided into two groups based on whether they underwent technically successful collateral embolization (UAE-SCE group) or failed collateral embolization (UAE-FCE group) in addition to UAE. The baseline characteristics and clinical outcomes including time for serum $\beta$-human chorionic gonadotropin ( $\beta-h C G$ ) levels normalization, blood loss, secondary anemia, and pelvic pain were compared between the two groups. The paired t test and Man Whitney test were used for comparisons of discrete and numerical variables, respectively.

Results: Collateral embolization was techinically successful in $16(66.7 \%, 16 / 24)$ patients and failed in the other 8 $(33.3 \%, 8 / 24)$ patients. There were no significant differences between the two groups in baseline characteristics. The mean blood loss and secondary anemia in the UAE-SCE group were significantly less than UAE-FCE group. No significant difference was found between the two groups in the mean time for $\beta$-hCG levels normalization and pelvic pain.

Conclusions: During the management of UAE combined with hysteroscopic curettage for CSP, additional embolization of non-gonadal collateral supplying GS during UAE is feasible and safe in patients with non-gonadal collateral supplying GS, and the additional embolization of the collateral may reduce blood bloss related to hysteroscopic curettage.
\end{abstract}

Keywords: Cesarean scar pregnancy, Collateral, Curettage, Intervention, Uterine artery embolization

\footnotetext{
* Correspondence: szt19641121@sina.com

Department of Interventional Radiology, Shandong Medical Imaging

Research Institute Affiliated to Shandong University, Jinan 250021, People's

Republic of China
}

(c) The Author(s). 2019 Open Access This article is distributed under the terms of the Creative Commons Attribution 4.0 International License (http://creativecommons.org/licenses/by/4.0/), which permits unrestricted use, distribution, and reproduction in any medium, provided you give appropriate credit to the original author(s) and the source, provide a link to the Creative Commons license, and indicate if changes were made. The Creative Commons Public Domain Dedication waiver (http://creativecommons.org/publicdomain/zero/1.0/) applies to the data made available in this article, unless otherwise stated. 


\section{Background}

Cesarean scar pregnancy (CSP) is a rare type of ectopic pregnancy defined as a gestational sac (GS) that implants at the site of a previous hysterotomy scar [1]. Currently, the diagnostic criteria of CSP are debated and still not standardized as patient presentations vary considerably, and the diagnosis of CSP made by ultrasound has been widely used.

Considering the risk of complications including uterine rupture, abnormal placentation, invasion into surrounding organs, massive vaginal bleeding and hypovolemic shock, pregnancy termination is generally recommended for patients with diagnosed CSP. In the documented treatment options for the management of CSP, uterine artery embolization (UAE) combined with another techniques including hysteroscopic resection, dilation and curettage, or curettage has been described as an efficient and safe treatment modality. In these combined techniques, the main goal of UAE was to occlude blood flow of bilateral uterine arteries by which to reduce high risk of vaginal hemorrhage related to the following curettage or hysteroscopic resection and accelerate the resolution of the gestational sac [2-4].

Although the bilateral uterine arteries provide the dominant uterine blood supply to uterine fibroid, recruitment of collateral supplying fibroid from the ovarian artery has been well documented in the literature. And it was also documented that failure to recognize and/or embolize the collaterals supplying fibroid during UAE was responsible for substandard clinical result $[5,6]$.

Similarly, from our experiences in UAE, the GS was supplied by collateral besides gonadal arteries (i.e., bilateral uterine arteries and ovarian arteries) in some CSP cases. To our knowledge, it was not documented the role of the non-gonadal collateral supplying GS in UAE.

Therefore, the purpose of the study was to assess the feasibility, safety and outcome of the embolization of non-gonadal collateral supplying GS in addition to UAE, followed by hysteroscopic curettage for the management of CSP.

\section{Methods}

\section{Patient selection}

Our hospital is the tertiary referral centre providing a range of subspecialty services for more than 60 hospitals in China. From January 2012 to September 2018, 481 patients with diagnosed CSP underwent UAE followed by hysteroscopic curettage in our hospital. In 24 of 481 $(5.0 \%, 24 / 481)$ patients, non-gonadal collaterals supplying GS were identified by arterial angiography performed immediately after UAE. These 24 patients underwent attempt collateral embolization in addition to UAE, and were included for retrospective analysis.
This study complies with current ethical consideration and was approved by our institutional ethical committee. The combined procedure of UAE and additional embolization of non-gonadal collateral supplying GS followed by hysteroscopic curettage was explained in full to each patient, and each patient gave an informed consent.

The diagnostic criteria for CSP included an increased serum $\beta$-human chorionic gonadotropin ( $\beta$-hCG) level (normal range less than $5 \mathrm{mIU} / \mathrm{mL}$ ), history of a prior cesarean scar, and ultrasound image that met the criteria proposed by Godin et al. [7], as follows: (i) absence of GS in uterine cavity and cervical canal; (ii) presence of a GS located at the anterior isthmus of uterus with or without cardiac activity; (iii) thinning or absence of myometrium between bladder and the GS (Fig. 1).

All the patients were hospitalized in the department of gynecology. The baseline characteristics of the patients including maternal age, time interval since last cesarean section, gestational age, GS diameter, myometrium thickness (the thickness of the myometrium between the GS and the bladder), and serum $\beta$-hCG level were recorded.

\section{Arterial embolization and hysteroscopic curettage}

The patients underwent UAE in our interventional department by two experienced interventional radiologists. Using Seldinger technique, the right common femoral artery access was achieved under local anesthesia. A 5-Fr Cobra catheter (Cordis, USA) was advanced distally to selectively catheterize the left internal iliac artery (IIA) negotiating with a hydrophilic guide-wire. The subsequent digital subtraction angiography (DSA) was performed at the IIA using $16 \mathrm{~mL}$ of nonionic contrast medium (Ultravist; Bayer, Germany) at a flow rate of 4 $\mathrm{mL} / \mathrm{s}$.

The DSA performed at the IIA was attempt to visualize the vascular anatomy of uterine artery, uterine perfusion, and the hypervascularity enhancement in GS region. When necessary, DSA of the IIA was performed with ipsilateral anterior oblique projection $\left(30^{\circ}\right)$ to reduce the imaging superimpositions and detect the origin of uterine artery. Then, a coaxial microcatheter (Stride 2.6 F, Asahi Intecc or Progreat 2.7 F, Terumo) was introduced coaxially into the uterine artery and advanced distally beyond the origin of the cervicovaginal branch with the digital roadmapping of the IIA. Selective uterine artery angiography was performed to ensure the trajectory of the uterine artery, uterine perfusion, and the hypervascularity enhancement in GS region. The subsequent embolization was performed by injecting the embolic agent slowly under continuous fluoroscopic guidance without reflux to undesired arteries. The embolic agents were 1000-1400 $\mu \mathrm{m}$ sized gelatin sponge particles (Alicon Co. Ltd., Hangzhou, China), which were absorbable 

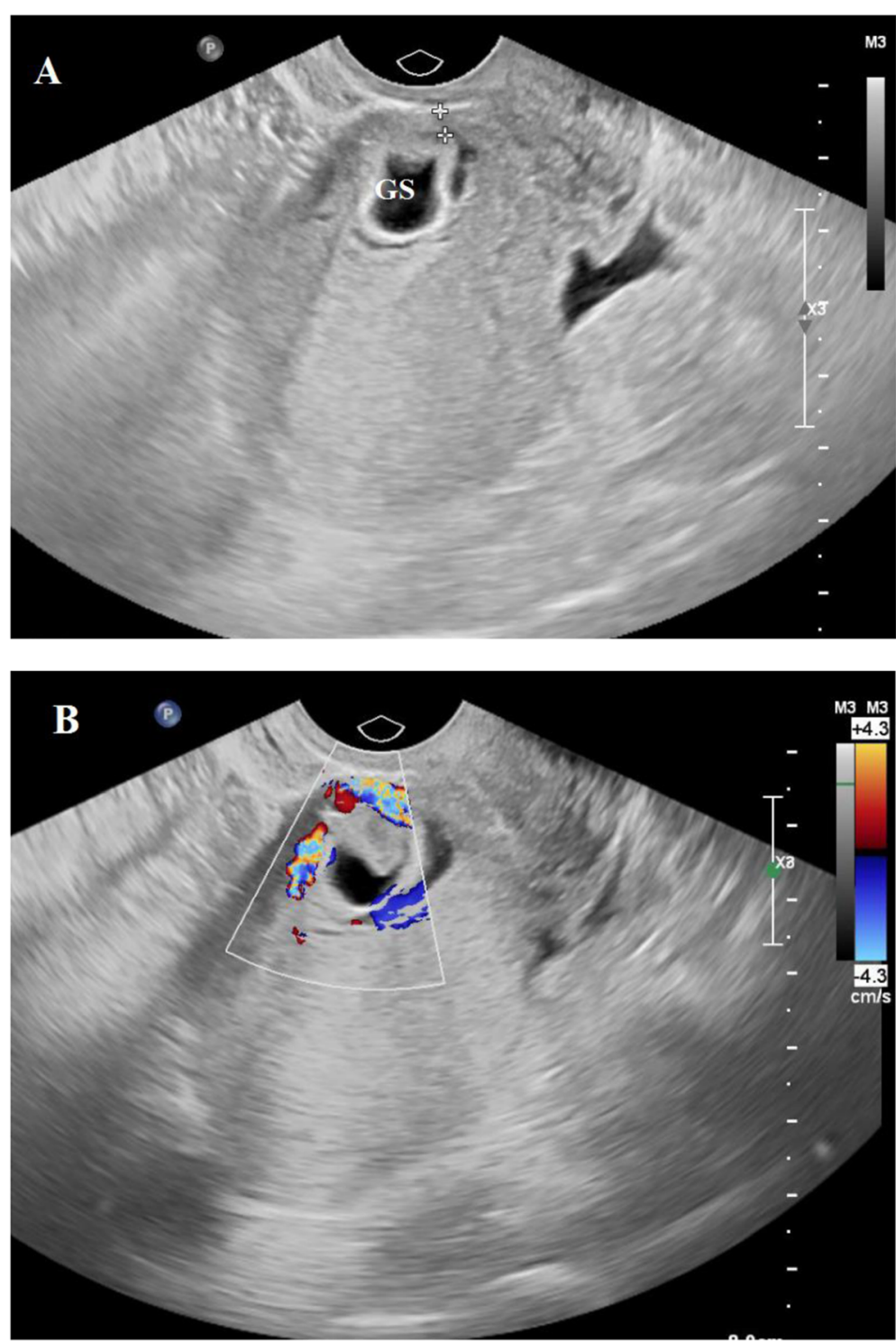

Fig. 1 Transabdominal ultrasound (a) of a patient with caesarean scar pregnancy showed an empty uterine cavity and cervical canal, and the gestational sac (GS) implanted at the site of a cesarean scar and surrounded by thin myometrium. The Doppler ultrasound (b) revealed the increased vascular flow surrounding the GS

agents. Each vial of gelatin sponge particles $(1 \mathrm{~mL})$ was mixed with $50 \mathrm{~mL}$ solution of contrast medium and saline solution at a 1:1 ratio. Embolization of the uterine artery was performed to the point of complete stasis of blood flow achieved in the ascending uterine artery.

Then postembolization angiongraphy at IIA after the removal of microcatheter was performed immediately to confirm the arterial occlusion of uterine artery and identify the possible presense of collateral supplying GS. The artery with the angiographic appearance of neovascularity and corresponding enhancement in the GS region was interpreted as a collateral supplying GS. Once a collateral supplying GS was identified, further selective microcatheterization and angiography was performed in the collateral to ensure the corresponding enhancement, followed by embolization with aforementioned gelatin sponge particles to the point of complete stasis of blood flow. After the embolization of the left uterine artery and collateral was finished, a Waltman loop was created on the Cobra catheter and the embolization of the right uterine artery and collateral was performed in the same way (Fig. 2). Analgesics and antiemetics were administered when needed.

After the embolization, the patient received $100 \mathrm{mg}$ of mifepristone per day for cervical preparation. The following removal of the GS using hysteroscopic curettage 

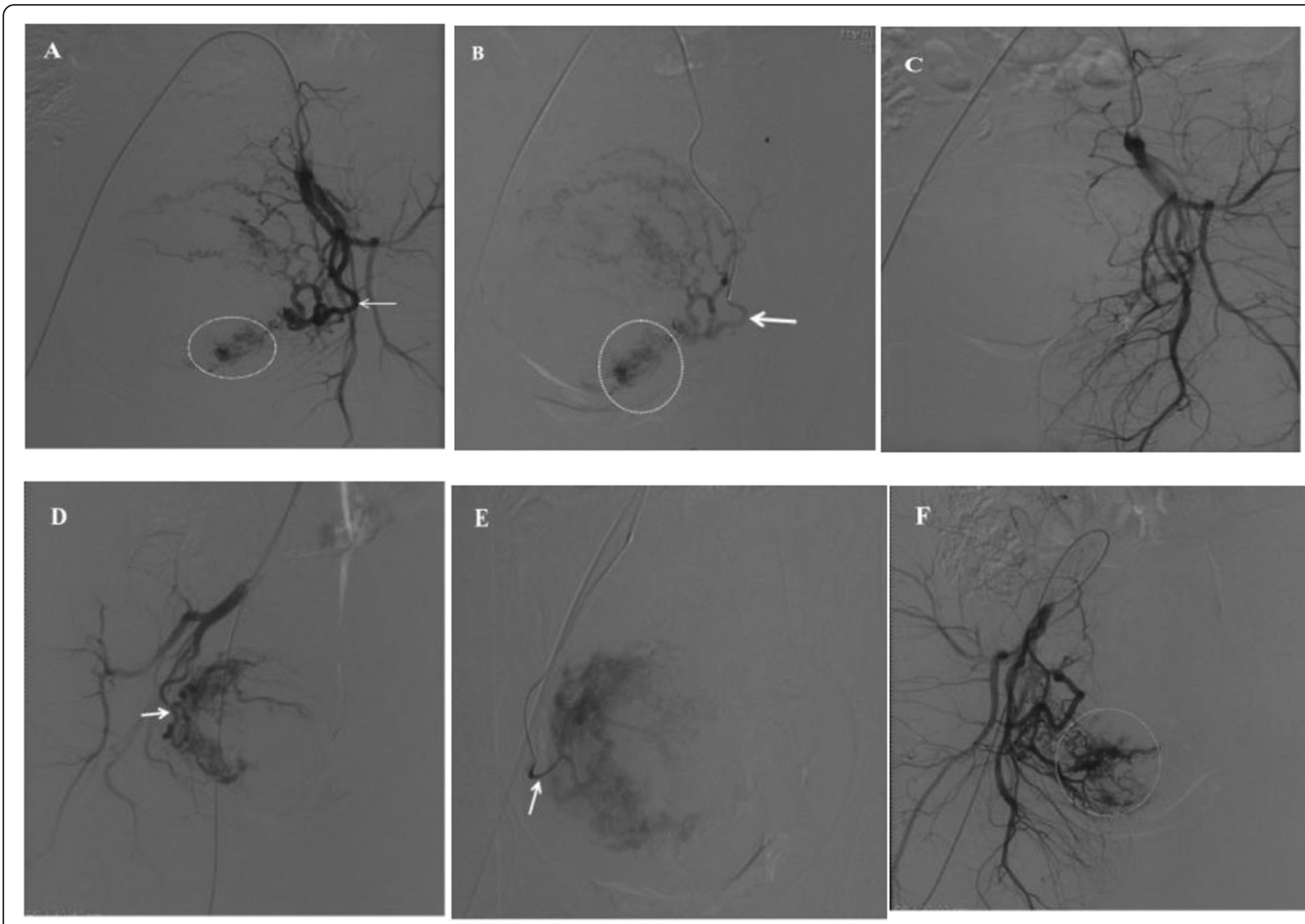

F
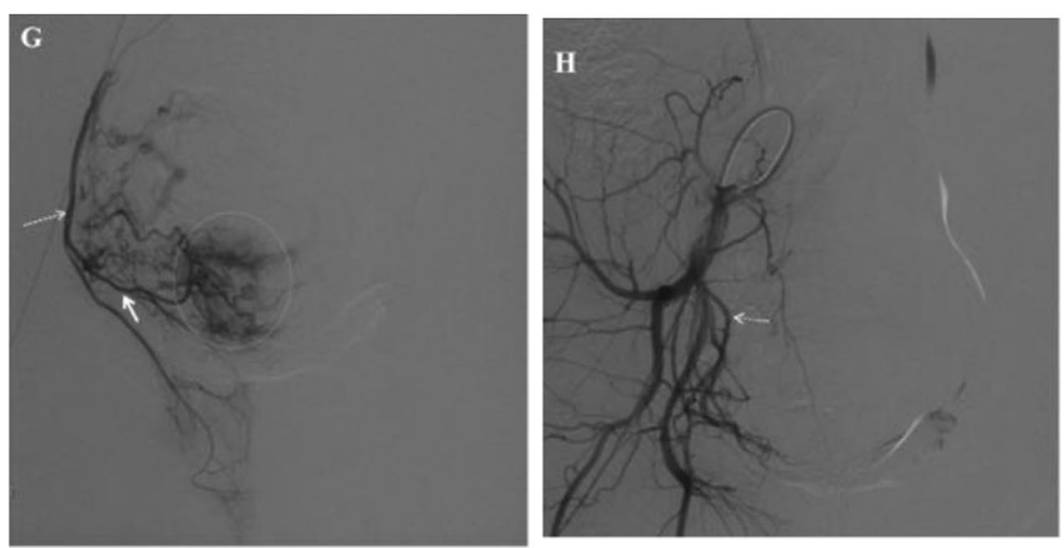

Fig. 2 (See legend on next page.) 
(See figure on previous page.)

Fig. 2 Representative angiographic images of a 32-year-old female patient suffering from cesarean scar pregnancy undergoing embolization of $>$ collateral supplying gestational sac (GS) in addition to uterine artery embolization. Angiography (a) of the left internal iliac artery (IIA) demonstrates the dilated left uterine artery (straight arrow) with typical tortuous trajectory and uterine perfusion, and a hypervascular gestational sac (dotted circle). Selective angiography (b) following microcatheterization of the left uterine artery (straight arrow) confirms the uterine perfusion and the hypervascular gestational sac (dotted circle). Angiography (c) of the left IIA performed after embolization of left uterine artery demonstrates the occlusion of left uterine artery and complete disappearance of uterine perfusion and hypervascularity enhancement in the GS region. Angiography (d) of the right IIA performed with ipsilateral anterior oblique projection $\left(30^{\circ}\right)$ demonstrates the dilated right uterine artery with typical tortuous trajectory and marked uterine perfusion. Selective angiography (e) following microcatheterization of the right uterine artery confirms the uterine perfusion. Angiography ( $\mathbf{f}$ ) of the right IIA performed after embolization of right uterine artery demonstrates the occlusion of right uterine artery, and the marked neovascularity and hypervascularity enhancement in the GS region (dotted circle). Selective angiography (g) following microcatheterization indicates the GS is supplied by a collateral (straight arrow) originating from the right internal pudendal artery (dotted arrow). The target collateral shows marked neovascularity and hypervascularity enhancement in the GS region (dotted circle). Angiography (h) of the right IIA performed after embolization of the target collateral demonstrates complete disappearance of hypervascularity enhancement in the GS region, without occlusion of the right internal pudendal artery (dotted arrow)

technique was conducted by experienced gynecologists within $48 \mathrm{~h}$ post-embolization. Under epidural anaesthesia, an operative hysteroscope with a 10-mm external diameter was placed inside the uterus following the cervical dilatation by Hegar dilators. Uterine distension was obtained using $5 \%$ glucose solution propelled by a uterine expansion instrument. Subsequently, an operative 26F hysteroscopic resectoscope with an electric wire loop electrode was introduced under transabdominal sonography guidance. The GS was pushed with the wire loop to expose the blood vessel bed of the implantation site. Then, the GS was removed using placenta forceps under direct vision. The following suction curettage with the wire loop was perfomed to clear the residual gestational tissue from the uterine wall. Coagulating the blood vessels with hysteroscopic rolling ball was used to control bleeding if required. This process continued until the gestational tissue was cleared completely and the myometrium was visualized (Fig. 3).

According to the protocols, ultrasonography was performed 3 days after the procedure, and serum $\beta$-hCG level was assessed before the procedure and every 3 days after procedure until discharge. Blood count was monitored before the procedure and $24 \mathrm{~h}$ after procedure. $\mathrm{Pa}-$ tients were discharged when (i) vaginal bleeding ceased or was mild; (ii) steadily decreasing in serum $\beta$-hCG levels was observed; and (iii) pelvic pain was absent. Follow-ups after discharge included repeat ultrasonic examination and serum $\beta$-hCG level measurement that were performed every 2 weeks until the serum $\beta$-hCG level returned to normal, and no residual GS was detected.

\section{Outcome evaluation}

The items evaluations were as follows: anatomic characteristics of the collateral supplying GS, technical success of collateral embolization, and clinical outcomes including time for $\beta$-hCG levels normalization and side effects.

Anatomic characteristics of the collateral supplying GS consisting of the origin of each collateral and whether unilateral or bilateral collaterals identified in each patient were recorded. Technical success of collateral embolization was considered when selective embolization in all identified collaterals supplying GS, and complete disappearance of uterine perfusion and hypervascularity enhancement in the GS region on post-embolization angiography at IIA were achieved. Side effects included post-embolization pain, the amount of intraoperative blood loss during curettage, secondary anemia, and whether need for additional blood transfusion or further hysterectomy or laparotomy.

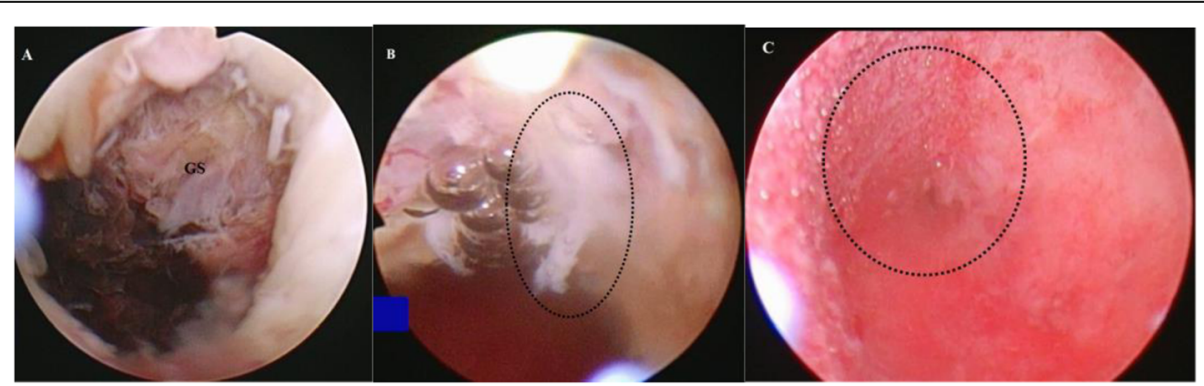

Fig. 3 Hysteroscopic management of cesarean scar pregnancy after uterine artery embolization. a Before hysteroscopic treatment, the gestational sac (GS) was visualized clearly. b After the GS was removed, only residual gestational tissue (dotted circle) was seen. c The myometrium (dotted circle) was visualized after the residual gestational tissue was cleared completely using suction curettage 
In order to assess the role of the additional collateral embolization, the included patients were divided into two groups based on whether they underwent technically successful collateral embolization (UAE-SCE group) or failed collateral embolization (UAE-FCE group) in addition to UAE. The baseline characteristics and clinical outcomes were compared between the two groups.

\section{Statistics}

The paired $t$ test and Man Whitney test were used for comparisons of discrete and numerical variables, respectively. All statistics were determined using SPSS (SPSS, Chicago, USA) version 22.0 and $P$ value of less than 0.05 was considered to be statistically significant.

\section{Results}

Technical and anatomic result

In the 24 patients included for evaluation, collaterals supplying GS were unilateral in 21 patients and bilateral in 3 patients, resulting in a total of 27 collaterals. As a result, most of the collaterals $(81.5 \%, 22 / 27)$ originated from the intern pudental arteries, and the remaining collaterals $(18.5 \%, 5 / 27)$ originated from the anterior division of IIAs.

Collateral embolization was techinically successful in $16(66.7 \%, 16 / 24)$ patients (UAE-SCE group), and failed in the other $8(33.3 \%, 8 / 24)$ patients (UAE-FCE group) due to the impossibility of selective catheterization because of the angled origins (in 6 patients) or small-sized orfice of the collaterals (in 2 patients).

\section{Clinical results}

The baseline characteristics of the 24 patients were shown in Table 1. There were no significant differences between the two groups in maternal age, gestational age, time interval between last cesarean section and the current pregnancy, GS diameter, the mean serum $\beta$-hCG levels, or myometrium thickness.

The clinical outcomes after CSP treatments are presented in Table 2. There was no significant difference between the two groups in the mean serum $\beta$-hCG levels on post-procedual 3rd day, and the mean time for $\beta$-hCG levels normalization. There were no significant differences between the two groups in slight pelvic pain. All the patients with slight pelvic pains recovered quickly after symptomatic treatments.

However, the mean blood loss in the UAE-SCE group was significantly less than UAE-FCE group $(P=0.025)$. The number of secondary anemia in the UAE-SCE group was also significantly less than UAE-FCE group $(P=0.01)$. As a result, among the 3 patients with secondary anemia to curettage in the UAE-FCE group, 1 patient received additional treatment with blood transfusion. No hysterectomies or laparotomies were needed for either of the two groups.

\section{Discussion}

The frequency of CSP has increased with the frequency of cesarean section in the recent decade, which might be due to the continuous increase in the cesarean deliveries and the improvement in ultrasound technique which enables early diagnosis of CSP [8]. The reported alternative treatments for CSP included laparotomy, surgical resection, hysteroscopic resection, UAE combined with other techniques including uterine dilation and curettage, systemic or local administration of methotrexate, or hysteroscopic curettage [3]. However, the optimal management for CSP remains unclear.

Although laparoscopic management for CSP has been described with the advantages of a high success rate and acceleration of time for $\beta$-hCG levels normalization. Due to the associated risk of massive intraoperative vaginal bleeding which required additional treatments, the use of single hysteroscopic management in terminating CSP is not adequate $[9,10]$. Thus, in the present study, UAE was incorporated as a preventive preceding method and performed prior to hysteroscopic curettage for the treatment of CSP. However, massive vaginal bleeding was still be occasionally encountered in patients undergoing the combined techniques. After a retrospective analysis, we found that unsuccessful occlusion of significant collaterals supplying the GS resulted in unsatisfactory

Table 1 Baseline characteristics of the 24 CSP patients with collateral supplying gestational sac

\begin{tabular}{llll}
\hline Characteristic & UAE-SCE group $(n=16)$ & UAE-FCE group $(n=8)$ & $p$ value \\
\hline Maternal age (years) & $32.44 \pm 5.60$ & $31.13 \pm 6.29$ & 0.725 \\
Time interval since last cesarean section (years) & $5.50 \pm 3.16$ & $4.75 \pm 3.20$ & 0.930 \\
Gestational age (days) & $54.50 \pm 15.00$ & $71.38 \pm 7.63$ & 0.136 \\
Gestational sac diameter $(\mathrm{mm})$ & $3.61 \pm 1.67$ & $5.66 \pm 1.02$ & 0.208 \\
Myometrium thickness $(\mathrm{mm})$ & $3.11 \pm 0.72$ & $3.09 \pm 0.86$ & 0.434 \\
Pre-procedural serum $\beta$-hCG level $(\mathrm{mIU} / \mathrm{mL})$ & $58,852.07 \pm 12,889.48$ & $59,607.57 \pm 23,137.26$ & 0.505 \\
\hline
\end{tabular}

The data are expressed as mean \pm standard deviation

CSP Cesarean Scar Pregnancy, UAE Uterine Artery Embolization, SCE Successful Collateral Embolization, FCE Failed Collateral Embolization, $\beta$ - $h$ CG $\beta$-human Chorionic Gonadotropin 
Table 2 Clinical patient outcomes

\begin{tabular}{llll}
\hline Characteristic & UAE-SCE group $(n=16)$ & UAE-FCE group $(n=8)$ & $p$ value \\
\hline Post-procedural serum $\beta$-hCG level $(\mathrm{mlU} / \mathrm{mL})$ & $9296.08 \pm 2425.87$ & $8921.17 \pm 4182.38$ & 0.740 \\
Time for $\beta$-hCG normalization (days) & $28.94 \pm 1.41$ & $27.88 \pm 1.86$ & \\
Side effects & & 1 & 0.574 \\
Pelvic pain ( $\mathrm{n})$ & 3 & $712.50 \pm 110.91$ & 0.705 \\
Amount of blood loss $(\mathrm{mL})$ & $78.75 \pm 26.63$ & 3 & 0.025 \\
Anemia $(\mathrm{n})$ & 0 & 0.01 &
\end{tabular}

The data of pelvic pain and anemia are listed as number, and the other data are expressed as mean \pm standard deviation

UAE Uterine Artery Embolization, SCE Successful Collateral Embolization, FCE Failed Collateral Embolization, $\beta$ - $h C G$-human Chorionic Gonadotropin

clinical results compared with successful occlusion of collaterals. Our finding was similar to previous study that reported nontreatment of the collateral supplying the fibroid during UAE for fibroid maybe associated with the clinical failure $[5,6,11-13]$.

In the present study, the collaterals supplying GS originated from the intern pudental arteries, or anterior division of IIAs. To avoid the possible reflux of embolic material and nontarget embolization of intern pudental arteries or other branches of IIAs, the microcatheter was advanced distally in the target collateral before the delivery of embolic material.

The arterial anotomy of pelvis is complex and variant. From our experiences, the use of the ipsilateral anterior oblique projection $\left(30^{\circ}\right)$ in the initial angiography of the IIA is helpful to identify/recognize the origins of the uterine artery, intern pudental artery, and the collateral supplying the GS.

It is challenging to selectively catheterize the collaterals supplying GS. The technical failure of collateral embolization was usually ascribed to the tortuosity or small diameter orfice of the target collaterals, which hampered the selective microcatheterization.

Additionally, UAE has been described to slightly reduce fertility and pregnancy rate which was possibly secondary to ovarian failure resulting from nontarget ovarian embolization via ovarian artery-to-uterine artery anastomoses [14-16]. In the present study, when selective embolization of uterine arteries and collaterals was performed, absorbable and large diameter embolic agents were used, so as to preserve the uterine and avoid nontarget embolization of ovarian arteries as much as possible.

The present study has several limitations. First, it was a retrospective study, so there may have been the possibility of selection bias, and the pre-procedural clinical and imaging data was incomplete. Second, there was only small number of patients included because of the low incidence of collaterals during the study period. Third, the added procedure time and radiation exposure due to the additional collateral embolization were not assessed.
The present study is somewhat limited by the small sample size and the feasibility of selective catheterization of collaterals supplying the GS. However, to the best of our knowledge, this is the first study to report on technical aspects as well as clinical outcome of embolization of non-gonadal collateral supplying GS in addition to $\mathrm{UAE}$, followed by hysteroscopic curettage for the management of CSP.

\section{Conclusions}

During the management of UAE combined with hysteroscopic curettage for CSP, additional embolization of nongonadal collateral supplying GS during UAE is feasible and safe in patients with non-gonadal collateral supplying GS, and the additional embolization of the collateral may reduce blood bloss related to hysteroscopic curettage.

\section{Abbreviations \\ CSP: Cesarean Scar Pregnancy; FCE: Failed Collateral Embolization; GS: Gestational Sac; IIA: Internal Iliac Artery; SCE: Successful Collateral Embolization; UAE: Uterine Artery Embolization; $\beta$-hCG: $\beta$-human Chorionic Gonadotropin}

\section{Acknowledgements}

The authors thank all patients for their participation.

\section{Authors' contributions}

ZTS and GDZ designed the study. ZTS was the principal investigator of the study. GDZ was the lead contributor in writing the manuscript. JJL helped analyse the data and contributed to writing the manuscript. JT is co-investigator of the study. LZ and DCW did the collecting data and interpreted the data. All authors read and approved the final manuscript.

\section{Funding}

This present research did not receive any specific grant from funding agencies in the public, commercial, or not-for-profit sectors.

\section{Availability of data and materials}

The datasets used and analysed during the current study are not publicly available, but are available from the corresponding author on reasonable request.

\section{Ethics approval and consent to participate}

The study complies with current ethical consideration and was approved by the institutional review board of Shandong medical imaging research institute and Shandong provincial hospital (decision number of our institutional ethics committee approval: JN-2012010038). All participants recruited in this study provided informed written consent. 


\section{Consent for publication}

Not applicable.

\section{Competing interests}

The authors declare that they have no competing interests.

Received: 24 June 2019 Accepted: 11 November 2019

Published online: 16 December 2019

\section{References}

1. Rotas MA, Haberman S, Levgur M. Cesarean scar ectopic pregnancies: etiology, diagnosis, and management. Obstet Gynecol. 2006;107(6):1373-81.

2. Tumenjargal A, Tokue H, Kishi H, Hirasawa H, Taketomi-Takahashi A, Tsushima Y. Uterine artery embolization combined with dilation and curettage for the treatment of cesarean scar pregnancy: efficacy and future fertility. Cardiovasc Intervent Radiol. 2018;41(8):1165-73.

3. Birch Petersen K, Hoffmann E, Rifbjerg Larsen C, Svarre NH. Cesarean scar pregnancy: a systematic review of treatment studies. Fertil Steril. 2016; 105(4):958-65.

4. Qiao B, Zhang Z, Li Y. Uterine artery embolization versus methotrexate for cesarean scar pregnancy in a Chinese population: a meta-analysis. J Minim Invasive Gynecol. 2016;23(7):1040-8

5. Pelage JP, Le Dref O, Soyer P, Jacob D, Kardache M, Dahan H, et al. Arteria anatomy of the female genital tract: variations and relevance to transcatheter embolization of the uterus. AJR Am J Roentgenol. 1999;172(4): 989-94.

6. Nikolic B, Spies JB, Abbara S, Goodwin SC. Ovarian artery supply of uterine fibroids as a cause of treatment failure after uterine artery embolization: a case report. J Vasc Interv Radiol. 1999;10(9):1167-70.

7. Godin PA, Bassil S, Donnez J. An ectopic pregnancy developing in a previous cesarean scar. Fertil Steril. 1997;67(2):398-400.

8. Liu G, Wu J, Cao J, Xue Y, Dai C, Xu J, et al. Comparison of three treatment strategies for cesarean scar pregnancy. Arch Gynecol Obstet. 2017;296(2): 383-9.

9. Deans R, Abbot J. Hysteroscopic management of cesarean scar ectopic pregnancy. Fertil Steril. 2010;93(6):1735-40.

10. Wang G, Liu X, Bi F, Yin L, Sa R, Wang D, et al. Evaluation of the efficacy of laparoscopic resection for the management of exogenous cesarean scar pregnancy. Fertil Steril. 2014;101(5):1501-7.

11. Yousefi S, Czeyda-Pommersheim F, White A, Banovac F, Hahn WY, Spies JB. Repeat uterine artery embolization: indications and technical findings. J Vasc Interv Radiol. 2006;17(12):1923-9.

12. Andrews RT, Bromley PJ, Pfister ME. Successful embolization of collaterals from the ovarian artery during uterine artery embolization for fibroids: a case report. J Vasc Interv Radiol. 2000;1 1(5):607-10.

13. Scheurig-Muenkler C, Poellinger A, Wagner M, Hamm B, Kroencke TJ. Ovarian artery embolization in patients with collateral supply to symptomatic uterine leiomyomata. Cardiovasc Intervent Radiol. 2011;34(6): 1199-207.

14. Keung JJ, Spies JB, Caridi TM. Uterine artery embolization: a review of current concepts. Best Pract Res Clin Obstet Gynaecol. 2018;46(1):66-73.

15. Pelage JP, Jacob D, Fazel A, Namur J, Laurent A, Rymer R, et al. Midterm results of uterine artery embolization for symptomatic adenomyosis:initial experience. Radiology. 2005;234(3):948-53.

16. Razavi MK, Wolanske KA, Hwang GL, Sze DY, Kee ST, Dake MD.

Angiographic classification of ovarian artery-touterine artery anastomoses: initial observations in uterine fibroid embolization. Radiology. 2002;224(3): 707-12.

\section{Publisher's Note}

Springer Nature remains neutral with regard to jurisdictional claims in published maps and institutional affiliations.

Ready to submit your research? Choose BMC and benefit from:
- fast, convenient online submission
- thorough peer review by experienced researchers in your field
- rapid publication on acceptance
- support for research data, including large and complex data types
- gold Open Access which fosters wider collaboration and increased citations
- maximum visibility for your research: over 100M website views per year
At BMC, research is always in progress.
Learn more biomedcentral.com/submissions

